\title{
Soft-material Robot for Ophthalmologic Surgery
}

\author{
Takahiro Kanno, ${ }^{1 *}$ Tomonori Kawakami, ${ }^{1}$ Minae Kawasaki, ${ }^{2}$ \\ Tomoya Furujo, ${ }^{2}$ Hiroshi Iida, ${ }^{3}$ Tetsuro Miyazaki, ${ }^{1}$ \\ Toshihiro Kawase, ${ }^{1,4}$ Kenji Kawashima, ${ }^{1}$ and Norihiko Ito $^{2}$ \\ ${ }^{1}$ Institute of Biomaterials and Bioengineering, Tokyo Medical and Dental University, \\ 2-3-10 Kanda-Surugadai, Chiyoda-ku, Tokyo 101-0062, Japan \\ ${ }^{2}$ Veterinary Medical Center, Tottori University, 4-101 Koyamacho-Minami, Tottori City 680-855, Japan \\ ${ }^{3}$ Iida Machinery Corporation, 3-19-17 Yahiro, Sumida-ku, Tokyo 131-0041, Japan \\ ${ }^{4}$ Institute of Innovative Research, Tokyo Institute of Technology, \\ 4259 Nagatsuta-cho, Midori-ku, Yokohama, Kanagawa 226-8503, Japan
}

(Received October 29, 2019; accepted February 4, 2020)

Keywords: surgical robot, soft robotics, pneumatic system, ophthalmology, pressure control

In this paper, a single-degree-of-freedom robot for assisting ophthalmologic surgery is proposed. A soft linear actuator drives the robot, and a needle attached to the tip punctures the cornea. The soft actuator has low friction and generates less stick slip, and there is no backlash since it does not require reduction gears. It can be controlled more precisely than conventional robots using electric motors or pneumatic cylinders. We conducted a cornea penetration experiment and measured the changes in intraocular pressure during intraocular injection using the proposed device.

\section{Introduction}

Ophthalmologic surgery is one of the most difficult surgical procedures. It requires the precise positioning of forceps within about $100 \mu \mathrm{m}$, which is smaller than the vibration of a normal human hand. Only a limited number of surgeons are available, and patients must wait for a long time to undergo such a surgery.

Robotic surgery is a possible solution to problems encountered in ophthalmologic surgery. Robots generally have high precision up to $10 \mu \mathrm{m}$ order. Surgical robots have filters to remove hand vibration. Da Vinci (Intuitive Surgical Inc.) ${ }^{(1)}$ and Senhance (TransEnterix Inc.) ${ }^{(2)}$ are the representative commercial robots for laparoscopic surgery.

Laparoscopic surgical robots have sufficient precision to conduct eye surgery. Culjat et al. carried out intraocular surgery using da Vinci. ${ }^{(3)}$ The robots designed specifically for ophthalmic surgery have also been developed. ${ }^{(4-8)}$ They are more compact than the robots for laparoscopy, and no interference between manipulators occurs in the narrow space around the eye.

Tadano et al. developed a laparoscopic surgical robot driven by pneumatic actuators, ${ }^{(9)}$ as opposed to conventional surgical robots that are driven by electric motors. The robot is compact

*Corresponding author: e-mail: kanno.bmc@tmd.ac.jp

https://doi.org/10.18494/SAM.2020.2668 
and lightweight, since pneumatic actuators output high forces without reduction gears. It is also possible to estimate external forces from the environment, such as those from suture threads and organs, utilizing the back drivability of pneumatic cylinders.

Actuator selection in designing a robot affects the positioning performance and the weight of the system. Positioning by electric motors is easy using high-ratio gears, although it tends to increase the cost for reducing the backlashes of gears. On the other hand, pneumatic cylinders have advantages in weight and cost. Pneumatic actuators, however, cause stick slip between the piston and the cylinder wall, making precise positioning difficult. Stick slip is reduced by using air-bearing cylinders, which increases the cost and decreases the energy efficiency.

A soft-material actuator is a possible alternative to conventional pneumatic actuators. It realizes both high precision and low cost while keeping the advantage of not requiring gears, which is a major advantage of pneumatic actuators. Conventional soft robots ${ }^{(10-14)}$ have been utilized for human robot interactions and the handling of complex-shape objects, which require safe or robust interactions but not precision. On the other hand, elastic actuators are utilized in the field of precision engineering. Kawashima et al. developed a precision stage using bellows actuators, ${ }^{(15)}$ realizing a $20 \mathrm{~nm}$ positioning error.

In this paper, we present a novel ophthalmologic surgery robot using a soft actuator. It is a single-degree-of-freedom injection device with a needle on its tip. The coil-reinforced-type linear soft actuator ${ }^{(16)}$ drives the needle. We conducted an experiment of inserting the needle into a porcine cornea and measured the transition of the intraocular pressure during insertion.

The rest of the paper is organized as follows. In Sect. 2, we describe the mechanical structure and control system of the proposed surgical robot. Experimental results and discussion are shown in Sect. 3. In Sect. 4, we conclude the paper.

\section{Materials and Methods}

\subsection{Mechanical structure of ophthalmologic surgery robot}

Figure 1 shows the overall structure of the proposed ophthalmologic surgery robot. A needle for ophthalmologic injection is attached to the tip of the linear soft actuator. The soft actuator

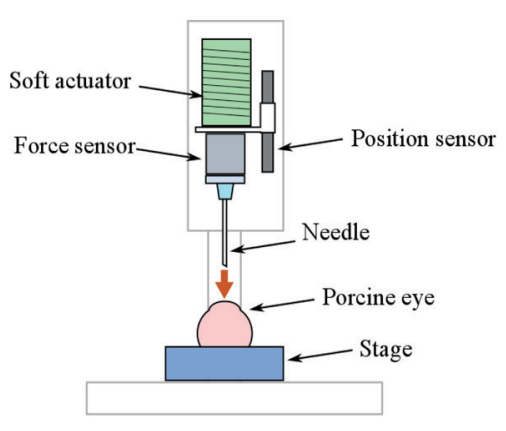

(a)

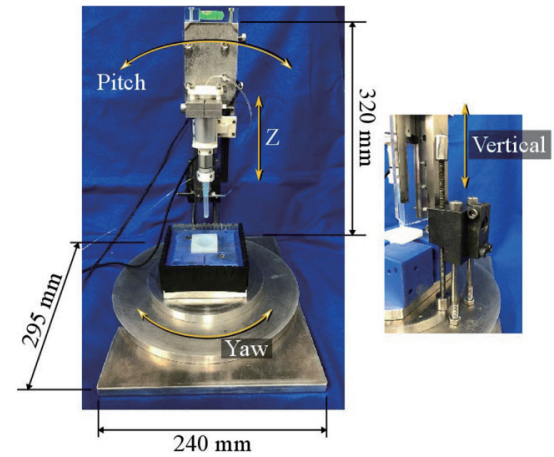

(b)

Fig. 1. (Color online) Ophthalmologic injection robot. (a) Schematic drawing. (b) Prototype. 
shown in Fig. 2 inside an aluminum pipe generates linear motion. It has a coil-reinforced structure, with the main body made of cylindrical silicone rubber (Smooth-sil 960, Smooth-on Inc.) and the stainless-steel coil (L066, Accurate Inc.) covering the rubber. The coil constrains the radial expansion of the rubber. The inner and outer diameters of the silicone cylinder are 16 and $18 \mathrm{~mm}$, respectively, and the length of the cylinder is $35 \mathrm{~mm}$. The wire diameter of the coil is $1 \mathrm{~mm}$. The soft actuator weighs $22 \mathrm{~g}$, while a pneumatic cylinder with the same inner diameter weighs $50 \mathrm{~g}$. The characteristics of the silicone material are shown in Table 1.

The maximum output force of the actuator is $60 \mathrm{~N}$ when the supply pressure is $0.3 \mathrm{MPa}$, which is much larger than the required force of about $1 \mathrm{~N}$. A smaller actuator is desirable for achieving a compact robot, although the current prototyping process using 3D-printed molds limits the minimum actuator size.

The soft actuator bends when external force or moment is applied radially. A linear guide (SSEB13, MISUMI) constrains the actuator motion to prevent the bending. The friction of the linear guide is ignorable compared with that of conventional pneumatic cylinders.

The intraocular pressure can be adjusted using a water tank and a precision regulator shown in Fig. 3. The linear-motion unit is mounted on the 4-degrees-of-freedom (DOF) base unit: yaw, vertical, pitch, and $Z$-axes. The base unit adjusts the location and orientation of the needle. These joints are passive and rotated manually.

\subsection{Measurement and control system}

Figure 4 shows a block diagram of the control system. The control system is a cascaded proportional-integral-differential (PID) control, in which an inner pressure control loop is included in an outer position control loop. The variables $q z$ and $q z_{r e f}$, and $P$ and $P_{\text {ref }}$ are the actuator displacements along the $Z$-axis in Fig. 1(b) and the air pressures in the actuator, respectively, where the subscript ref denotes the reference value. Control gains are defined in Table 2.

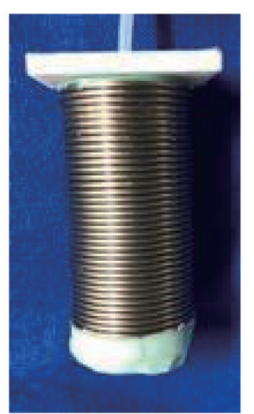

Fig. 2. (Color online) Soft actuator.

Table 1

Characteristics of silicone rubber. ${ }^{(17)}$

\begin{tabular}{lc}
\hline Shore hardness (A) & 60 \\
\hline Specific gravity (g/cc) & 1.25 \\
\hline Elongation at break (\%) & 270 \\
\hline Tensile strength (psi) & 650 \\
\hline
\end{tabular}

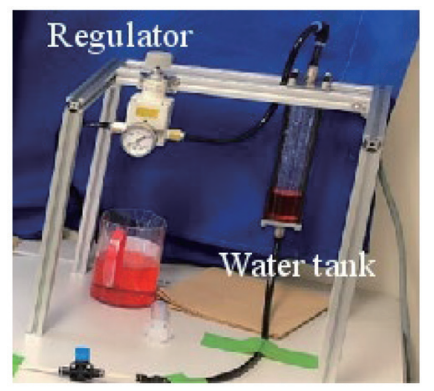

Fig. 3. (Color online) Intraocular pressure adjusting unit. 


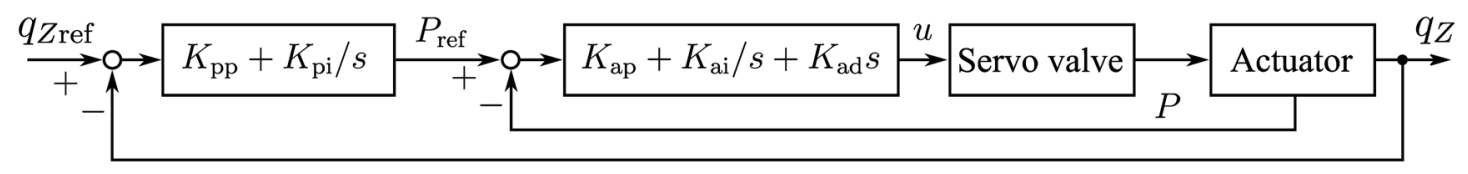

Fig. 4. Block diagram of position control.

Table 2

Control gains.

\begin{tabular}{lll}
\hline Proportional gain for position $(\mathrm{kPa} / \mathrm{mm})$ & $K_{p p}$ & 0.1 \\
\hline Integral gain for position $(\mathrm{kPa} \cdot \mathrm{s} / \mathrm{mm})$ & $K_{p i}$ & 100 \\
\hline Proportional gain for pressure $(\mathrm{V} / \mathrm{kPa})$ & $K_{a p}$ & 0.04 \\
\hline Integral gain for pressure $(\mathrm{V} / \mathrm{kPa} \cdot \mathrm{s})$ & $K_{a i}$ & 1.0 \\
\hline Differential gain for pressure $(\mathrm{V} \cdot \mathrm{s} / \mathrm{kPa})$ & $K_{a d}$ & 0.025 \\
\hline
\end{tabular}

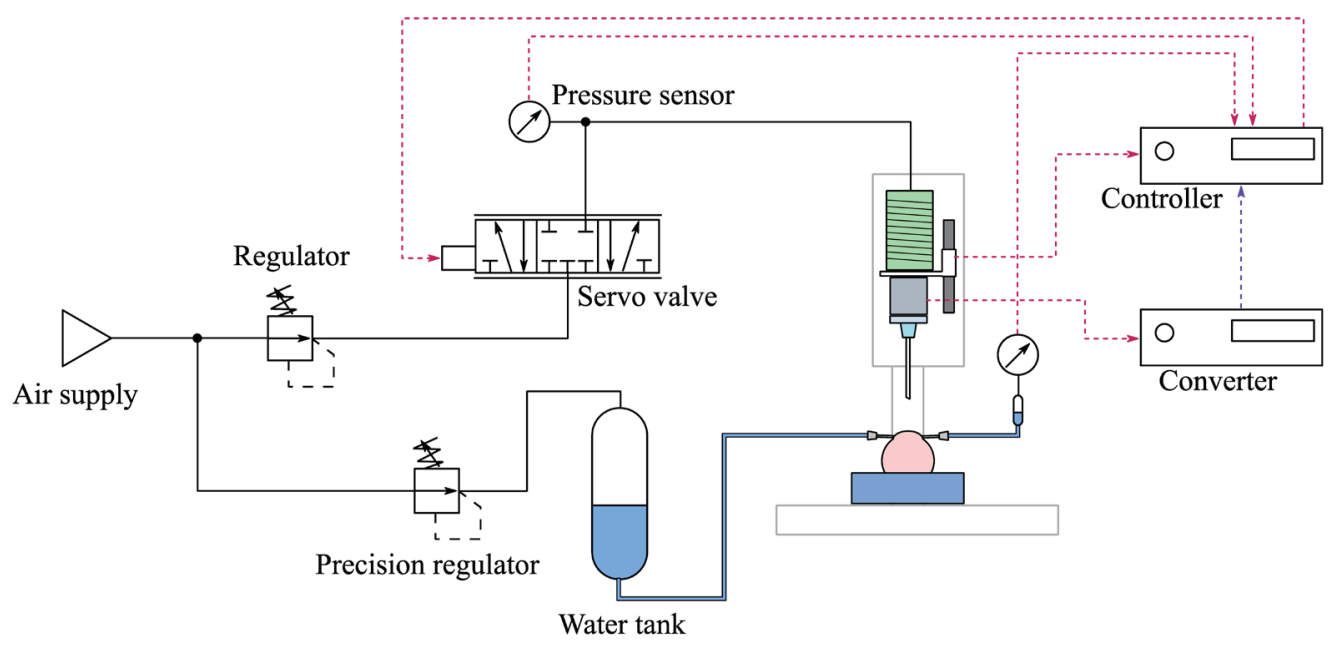

Fig. 5. (Color online) Control system setup.

Figure 5 shows the electric and pneumatic circuits. The pressure is controlled using a signal measured by a pressure sensor (PSE540, SMC Corp.) acquired via a 16-bit AD converter (AI-1616L, Contec Co., Ltd.). The position is controlled using a magnetic linear encoder (AS5311, ams AG) with a resolution of $2 \mu \mathrm{m}$, which is attached in combination with a magnetic scale (LMS-I1L1000-W10-A10-K, Bogen Electric GmbH). The magnetic pole pitch of the scale is $1 \mathrm{~mm}$. It is a noncontact encoder based on a hall sensor technology. The gap between the sensor chip and the scale is kept constant by the mechanism using the linear guide.

We also introduced a sensor to measure the intraocular pressure for monitoring. It is a precision pressure sensor (ZSE30AF-01-C-MG-X580, SMC Corp.) with the measurement range from -10 to $+10 \mathrm{kPa}$. A force torque sensor (BL NANO, BL-Autotec Co., Ltd.) between the actuator and the needle measures the contact force. The actuator automatically reduces the velocity when the contact between the needle and the cornea is detected by the force torque sensor. The sampling time of the control loop is $1 \mathrm{~ms}$. The control gains are shown in Table 2 . 


\subsection{Experimental protocol}

In this study, we measured the transition of the intraocular pressure during the insertion of a needle into a porcine cornea. A porcine eye is placed on the stage centered on the base unit as shown in Fig. 6. A transparent ring and anchor screws fix the eye. We inserted two cannulas: one for reflux and intraocular pressure adjustment, and the other for intraocular pressure measurement.

In the experiment, the motion of the robot is given as follows:

Step 1: Approach at $V_{1} \mathrm{~mm} / \mathrm{s}$ until the needle touches the cornea.

Step 2: Change the needle velocity to $V_{2}\left(<V_{1}\right)$ and moves for $L \mathrm{~mm}$ to penetrate the cornea.

Step 3: Stop for $T \mathrm{~s}$ in the anterior chamber.

Step 4: Return to the initial position at $V_{3} \mathrm{~mm} / \mathrm{s}$.

In Step 1, the system detects the contact when the measurement of the force detected by the sensor exceeds a threshold of $0.1 \mathrm{~N}$. The entire procedure is shown in Fig. 7, and the control parameters are shown in Table 3.

\section{Results and Discussion}

Figure 8 shows the experimental results. The upper, middle, and lower plots present the actuator displacement, the force measured by the force sensor, and the intraocular pressure, respectively. From this figure, the overall position tracking performance is good, even when it is driven by a soft actuator.

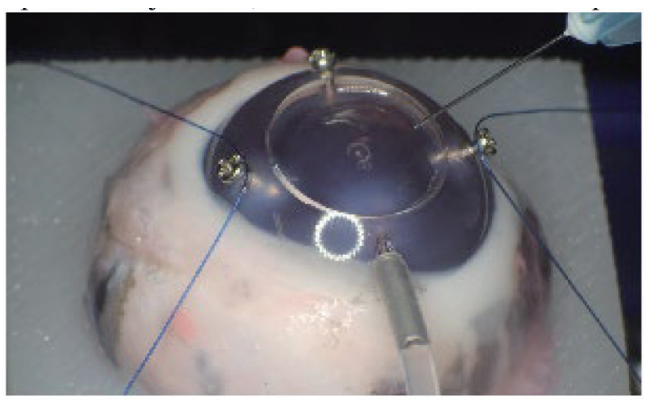

Fig. 6. (Color online) Experimental setup.
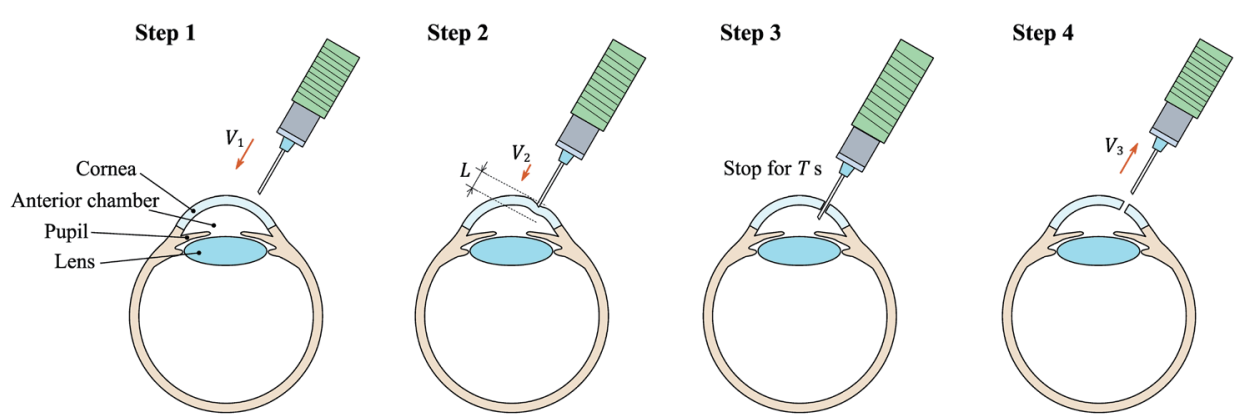

Fig. 7. (Color online) Needle insertion procedure. 
Table 3

Needle insertion parameters.

\begin{tabular}{lc}
\hline$V_{1}(\mathrm{~mm} / \mathrm{s})$ & 2.0 \\
\hline$V_{2}(\mathrm{~mm} / \mathrm{s})$ & 0.5 \\
\hline$V_{3}(\mathrm{~mm} / \mathrm{s})$ & 1.0 \\
\hline$L(\mathrm{~mm})$ & 3.0 \\
\hline$T(\mathrm{~s})$ & 10.0 \\
\hline
\end{tabular}

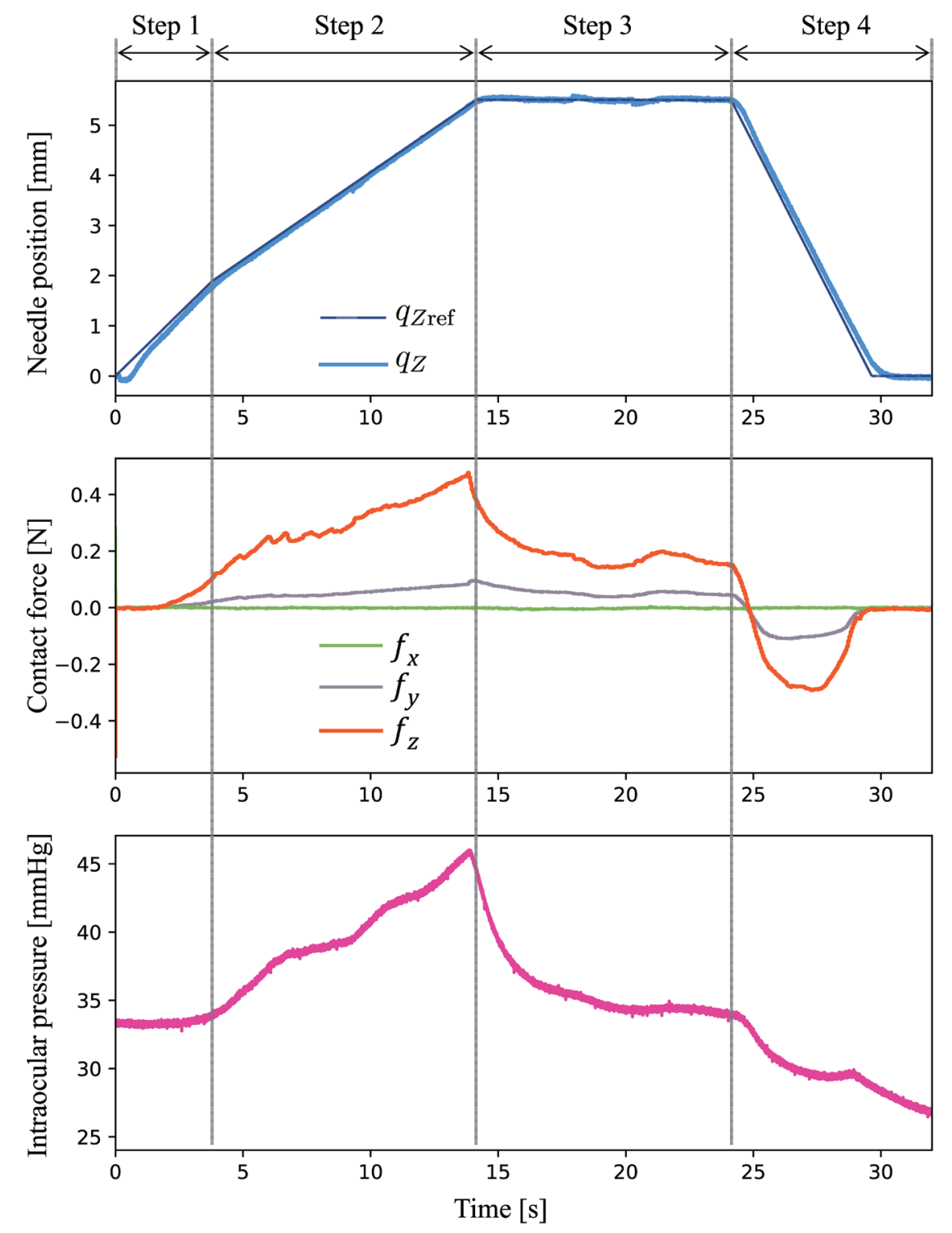

Fig. 8. (Color online) Experimental results.

When the needle advanced about $2 \mathrm{~mm}$, the force measurement along the $Z$-axis $\left(f_{Z}\right)$ exceeded the threshold, and the needle slowed down. During Step 2, the intraocular pressure continued to increase from 33 to $46 \mathrm{mmHg}$, since the eyeball was being compressed by the 
pushing force from the needle. In Step 3, both the force and the intraocular pressure decreased, although the needle was in a stationary state. This was because the shape of the eyeball gradually recovered after the needle completely penetrated the cornea.

The change in intraocular pressure was large, even though the pressure was adjusted by the regulator. This is due to the mechanical deadband of the regulator. The range within which pressure can be adjusted by the regulator ranged from 0 to $0.2 \mathrm{MPa}(1500 \mathrm{mmHg})$, while the pressure change in this experiment was less than $1 \%$ of the range of the regulator. To reduce the pressure and damage to the eye components, it is desired to introduce a pressure controller using a precision pressure sensor.

The positioning performance is important for precise surgery. The steady-state positioning error shown in Step 3 in Fig. 8 is $0.05 \mathrm{~mm}$. It is similar to that of the conventional robot driven by pneumatic cylinders. ${ }^{(18)}$ The desired precision is $0.1 \mathrm{~mm}$, considering the future application of the robot to retinal vessel cannulation surgery, where the diameter of the target blood vessel is about $0.1 \mathrm{~mm}$. $^{(19)}$

It seems enough for the proposed robot to conduct cannulation, although a more precise robot is desirable considering a safety factor. The control performance should be improved to make full use of the frictionless feature of the soft actuator. Besides the dynamic modeling of the robot and the feedforward control, a better sensor system, such as a laser encoder and low-noise electric circuits to increase the feedback gains, will be necessary to improve the positioning performance. It is also necessary to evaluate the precision when the robot is extended to multiple DOFs.

\section{Conclusions}

We proposed an ophthalmologic injection device. A linear soft actuator drives the injection needle. The soft actuator has a high positioning ability since it has no friction or backlash. We confirmed the good position control performance of the device achieved by introducing a cascaded pneumatic control system. An intraocular pressure adjustment device with a water reservoir and a precision air regulator is also developed.

Using the proposed system, we acquired the initial data on the dynamic transition of intraocular pressure. The intraocular pressure increased by about $20 \mathrm{mmHg}$, and we found the necessity to use the feedback control system to regulate the intraocular pressure to reduce damage to the eye components during an eye surgery.

\section{References}

1 G. Guthart and J. Salisbury: Proc. IEEE ICRA 2000 (2000) 618. https://doi.org/10.1109/ROBOT.2000.844121

2 F. Fanfani, G. Monterossi, A. Fagotti, C. Rossitto, S. G. Alletti, B. Costantini, V. Gallotta, L. Selvaggi, S. Restaino, and G. Scambia: Surg. Endosc. 30 (2016) 215. https://doi.org/10.1007/s00464-015-4187-9

3 M. Culjat, A. Tsirbas, A. Gupta, and S. D. Schwartz: Retina 28 (2008) 1. https://doi.org/10.1097/ IAE.0b013e318068de46.

4 K. W. Grace, J. E. Colgate, M. Glucksberg, and J. H. Chun: Proc. IEEE ICRA 1993, 630.

5 P. S. Jensen, K. W. Grace, R. Attariwala, J. E. Colgate, and M. R. Glucksberg: Graefes. Arch. Clin. Exp. Ophthalmol. 235 (1997) 11. https://doi.org/10.1007/bf01880668 
6 T. Ueta, Y. Yamaguchi, Y. Shirakawa, T. Nakano, R. Ideta, Y. Noda, A. Morita, R. Mochizuki, N. Sugita, M. Mitsuishi, and Y. Tamaki: Ophthalmology 116 (2009) 8. https://doi.org/10.1016/j.ophtha.2009.03.001

7 W. Wei, R. Goldman, N. Simaan, H. Fine, and S. Chang: Proc. IEEE ICRA 2007, 3389.

8 A. Gijbels, E. Vander Poorten, B. Gorissen, A. Devreker, P. Stalmans, and D. Reynaerts: IEEE RAS \& EMBS BioRob 2014, 144.

9 K. Tadano, K. Kawashima, K. Kojima, and N. Tanaka: JRM 22 (2010) 2. https://doi.org/10.20965/jrm.2010. p0179

10 J. Kim, A. Alspach, and K. Yamane: IEEE/RSJ IROS 2015, 2419.

11 P. Polygerinos, S. Lyne, Z. Wang, L. F. Nicolini, B. Mosadegh, G. M. Whitesides, and C. J. Walsh: IEEE/RSJ IROS 2013, 1512.

12 D. Rus and M. T. Tolley: Nature 521 (2015) 467.

13 F. Ilievski, A. D. Mazzeo, R. F. Shepherd, X. Chen, and G. M. Whitesides: Angew. Chem. Int. Ed. 50 (2011) 1890. https://doi.org/10.1002/anie.201006464

14 M. T. Tolley, R. F. Shepherd, B. Mosadegh, K. C. Galloway, M. Wehner, M. Karpelson, R. J. Wood, and G. M. Whitesides: Soft Rob. 1 (2014) 3. https://doi.org/10.1089/soro.2014.0008

15 K. Kawashima, T. Arai, K. Tadano, T. Fujita, and T. Kagawa: Precis. Eng. 34 (2010) 3. https://doi.org/10.1016/ j.precisioneng.2010.02.005.

16 T. Kanno, S. Ohkura, O. Azami, T. Miyazaki, T. Kawase, and K. Kawashima: Appl. Sci. 9 (2019) 2109. https:// doi.org/10.3390/app9102109

17 Smooth-On Inc. https://www.smooth-on.com/products/smooth-sil-960/ (accessed September 2019)

18 D. Haraguchi, T. Kanno, K. Tadano, and K. Kawashima: IEEE/ASME Trans. Mechatron. 20 (2015) 3. https:// doi.org/10.1109/TMECH.2015.2415838

19 Y. Ouyang, Q. Shao, D. Scharf, A. M. Joussen, and F. M. Heussen: Graefes. Arch. Clin. Exp. Ophthalmol. 253 (2015) 499. https://doi.org/10.1007/s00417-014-2715-2

\section{About the Authors}

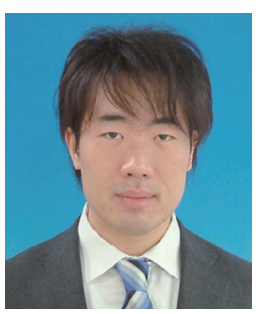

Takahiro Kanno received his doctoral degree in engineering from the Department of Mechanical Engineering and Science at Kyoto University in 2013. In 2013, he worked as a postdoctoral researcher in Precision and Intelligence Laboratory, Tokyo Institute of Technology. From 2013 to 2019, he then worked as an assistant professor at Tokyo Medical and Dental University. Since June 2019, he has been an associate professor there. His research interests include medical robotics, control engineering, and teleoperation. (kanno.bmc@tmd.ac.jp)

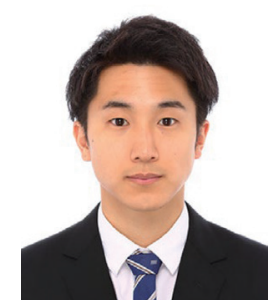

Tomonori Kawakami received his B.S. degree from the Department of Mathematics at Waseda University in 2017 and his M.S. degree in engineering from the Department of Health Sciences and Biomedical Engineering at Tokyo Medical and Dental University in 2019. (ktomonori40@gmail.com) 


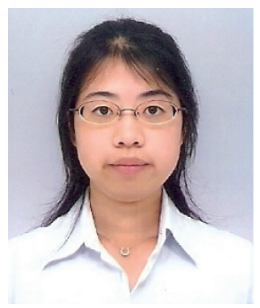

Minae Kawasaki received her BVSc degree from the Faculty of Veterinary Science at the University of Sydney, Australia, in 2012. She started her veterinary career in general practices, where she developed her interest in ophthalmology. She then moved to Tottori University as a project researcher and was involved in collaborative research studies on veterinary ophthalmology. She is currently enrolled in a $\mathrm{PhD}$ program and also works as a clinical assistant in ophthalmology service at Tottori University Veterinary Medical Center. She is interested in all aspects of small animal internal medicine, particularly ophthalmology. (i002tm@yamaguchi-u.ac.jp)

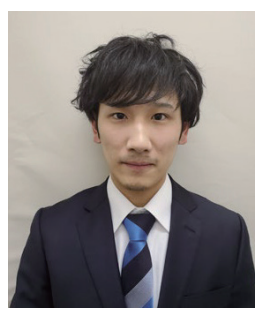

Tomoya Furujo received his B.S. degree from the Faculty of Agriculture at Tottori University. His research interests are in veterinary medicine and ophthalmology. (furrujo0724@hotmail.co.jp)

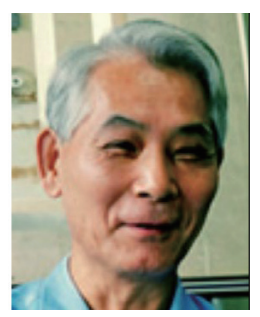

Hiroshi Iida is the Chief Executive Officer (CEO) of IIDA Machinery Corporation. His research interest is in the high-efficiency production of metal parts. He is developing and manufacturing medical devices, deep drawing products, and agricultural machines.

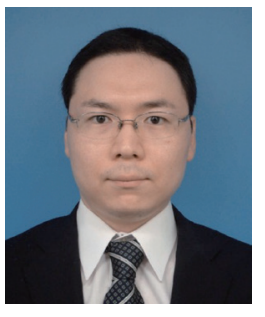

Tetsuro Miyazaki received his doctoral degree in engineering from the Department of Mechanical Sciences and Engineering at Tokyo Institute of Technology in 2014. He worked as a research assistant from 2014 to 2015 and an assistant professor from 2015 to 2017 at Yokohama National University. Since April 2017, he has been an assistant professor at the Institute of Biomaterials and Bioengineering at Tokyo Medical and Dental University. His research interests are in mechanical engineering, control engineering, power-assistive devices, and medical welfare robotics.

(tmiyazaki.bmc@tmd.ac.jp)

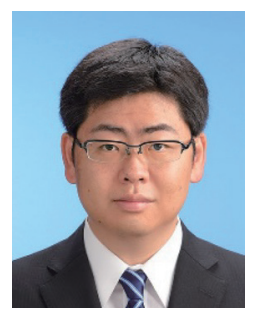

Toshihiro Kawase received his B.S., M.S., and Ph.D. degrees from Tokyo Institute of Technology, Tokyo, Japan in 2007, 2009, and 2012, respectively. He was a research fellow at the Research Institute of National Rehabilitation Center for Persons with Disabilities from 2012 to 2015, and worked as a postdoctoral fellow and a specially appointed assistant professor at Tokyo Institute of Technology from 2015 to 2017 . He is currently an assistant professor at Tokyo Medical and Dental University and Tokyo Institute of Technology. His research interests include rehabilitation robotics, medical robots, and biological signal processing. (kawase.bmc@tmd.ac.jp) 


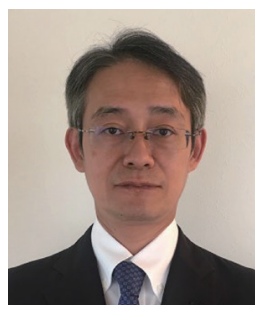

Kenji Kawashima received his doctoral degree in engineering from the Department of Control Engineering at Tokyo Institute of Technology in 1997. From 1997 to 2000, he worked as a research assistant at the Tokyo Metropolitan College of Technology. He then worked as an associate professor in the Precision and Intelligence Laboratory at Tokyo Institute of Technology. Since April 2013, he has been a professor at the Institute of Biomaterials and Bioengineering at Tokyo Medical and Dental University. His research interests include medical robotics, control engineering, and fluid measurement and control. (kkawa.bmc@tmd.ac.jp)

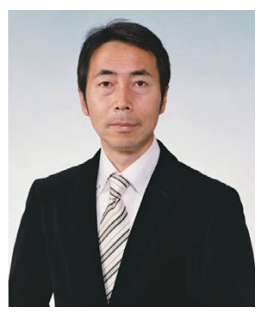

Norihiko Ito received his doctoral degree in medicine from the School of Medicine at Yokohama City University in 1999. From 2011 to 2013, he worked as an associate professor in the Center for Biological Resources and Informatics at Tokyo Institute of Technology. Since 2013, he has been an associate professor in Animal Medical Center at Tottori University. His research interests are in ophthalmology and medical robotics.

(taro@tottori-u.ac.jp) 